\title{
Mitogen-Activated Protein Kinase Is a Functional Component of the Autonomous Circadian System in the Suprachiasmatic Nucleus
}

\author{
Makoto Akashi, ${ }^{1 *}$ Naoto Hayasaka, ${ }^{3 *}$ Shin Yamazaki, ${ }^{4}$ and Koichi Node ${ }^{2}$ \\ Departments of ${ }^{1}$ Vascular Failure Research and ${ }^{2}$ Cardiovascular and Renal Medicine, Faculty of Medicine, Saga University, Saga 849-8501, Japan, \\ ${ }^{3}$ Department of Anatomy and Neurobiology, Kinki University School of Medicine, Osaka-Sayama, Osaka 589-8511, Japan, and ${ }^{4}$ Department of Biological \\ Sciences, Vanderbilt University, Nashville, Tennessee 37235
}

The suprachiasmatic nucleus (SCN) is the master circadian pacemaker driving behavioral and physiological rhythms in mammals. Circadian activation of mitogen-activated protein kinase [MAPK; also known as ERK (extracellular signal-regulated kinase)] is observed in vivo in the SCN under constant darkness, although the biological significance of this remains unclear. To elucidate this question, we first examined whether MAPK was autonomously activated in ex vivo SCN slices. Moreover, we investigated the effect of MAPK inhibition on circadian clock gene expression and neuronal firing rhythms using SCN-slice culture systems. We show herein that MAPK is autonomously activated in the SCN, and our data demonstrate that inhibition of the MAPK activity results in dampened rhythms and reduced basal levels in circadian clock gene expression at the SCN single-neuron level. Furthermore, MAPK inhibition attenuates autonomous circadian neuronal firing rhythms in the SCN. Thus, our data suggest that light-independent MAPK activity contributes to the robustness of the SCN autonomous circadian system.

Key words: circadian rhythms; ERK; suprachiasmatic nucleus; tissue culture; transcription; transgenic

\section{Introduction}

Almost all organisms on earth exhibit circadian rhythms and, therefore, the circadian clock is one of the universal biological systems (Reppert and Weaver, 2002). The circadian clock oscillates with an endogenous period, which is synchronized to the $24 \mathrm{~h}$ light/dark cycle in nature. The molecular oscillator that generates the clock consists of interconnected transcription-translation feedback loops (Dunlap, 1999). Circadian oscillators are cell-autonomous, and exist in most types of cells, including cultured cells (Schibler and Sassone-Corsi, 2002). It is thought that peripheral timekeepers are governed by signals produced by the suprachiasmatic nucleus (SCN), a defined pair of cell clusters in the anteroventral hypothalamus (Young and Kay, 2001). The SCN circadian clock is more robust and sustainable compared with peripheral clocks (Yamazaki et al., 2000), indicating that the SCN has some specific mechanism(s) for more persistent rhythms.

\footnotetext{
Received July 27, 2007; revised Feb. 12, 2008; accepted Feb. 12, 2008

This work was supported by fellowships from the Japan Society for the Promotion of Science and National Institutes of Health Grant NS05128 (S.Y.). We thank Hajime Tei, Rika Numano, Mamoru Nagano, Yoko Hatta-Ohashi, Masayuki lkeda, and Wataru Nakamura for technical advice; Hitomi Maeyama, Rie Ichiyama, Yuko Tsuboi, and Asuka Tsugitomi for their expert technical assistance; and Sato Honma for help and discussions. Per2-luciferase knock-in mice were kindly provided by Joseph Takahashi. We express our great appreciation to Tetsuaki Hirase, Teruo Inoue, and Yasufumi Shigeyoshi for general support.

${ }^{*}$ M.A. and N.H. contributed equally to this work.

Correspondence should be addressed to Makoto Akashi, Department of Vascular Failure Research, Saga University Faculty of Medicine, 5-1-1 Nabeshima, Saga 849-8501, Japan. E-mail: akashima@med.saga-u.ac.jp.

D01:10.1523/JNEUROSCI.3410-07.2008

Copyright $\odot 2008$ Society for Neuroscience $\quad$ 0270-6474/08/284619-05\$15.00/0
}

Previous studies have reported that transient and strong activation of mitogen-activated protein kinase [MAPK; the classical MAP kinase, also known as the extracellular signal-regulated kinase (ERK)] is induced in the SCN by a light pulse, suggesting that MAPK activation is an essential event for light entrainment of the circadian clock (Obrietan et al., 1998; Wang and Sehgal, 2002; Coogan and Piggins, 2004). However, many studies have demonstrated in rodents that circadian activation of MAPK is observed in the SCN even under constant darkness (Coogan and Piggins, 2004), although the biological significance of this remains unclear. We show herein that the autonomous activation of MAPK is indispensable for persistent circadian oscillations in the SCN clock.

\section{Materials and Methods}

Generation of transgenic mice. Transgenic mice were generated by using a linearized and gel-purified brain and muscle Arnt-like protein 1 (hBmall) (-3.5 kbp)::luciferase) (LUC) fragment. Transgenic animals were identified by genotyping tail genomic DNA samples by PCR. All experiments were conducted following the guidelines for the care and use of laboratory animals of Saga University Graduate School of Medicine, Kinki University School of Medicine, and Vanderbilt University.

Explant cultures and bioluminescence measurement. Coronal brain slices including the SCN (300-400 $\mu \mathrm{m}$ thickness) were prepared from adult transgenic mice, adult knock-in mice, or 2-week-old Wistar rats. Paired SCNs were excised and explanted from coronal brain sections and placed on a culture membrane (Millicell-CM, PICM030-50; Millipore, Billerica, MA) in a covered and sealed Petri dish. Bioluminescence was measured with a photomultiplier tube (Kronos, ATTO, or LM2400; Hamamatsu, Bridgewater, NJ) or a luminescence microscope optimized 
for live cell imaging (LV200; Olympus, Tokyo, Japan). The data sets were detrended by subtracting the $24 \mathrm{~h}$ running average from the raw data.

Immunohistochemistry. The slices were cultured for 1 week before fixation. They were immersed in $4 \%$ paraformaldehyde for $2 \mathrm{~d}$ at $4^{\circ} \mathrm{C}$. After rinsing, the slices were incubated for $3 \mathrm{~d}$ at $4^{\circ} \mathrm{C}$ in phospho-p44/42 MAP kinase antibody (Cell Signaling Technology, Danvers, MA) diluted 1:1500 in PBS containing 0.3\% Triton $\mathrm{X}-100$. The slices were rinsed and incubated for $24 \mathrm{~h}$ at $4^{\circ} \mathrm{C}$ in Fluorophor-labeled goat antirabbit IgG antibody (Alexa Fluor 488; Invitrogen, Eugene, OR) diluted 1:1000 in PBS. After rinsing, the slices were embedded in gel/mount (Biomeda, Foster City, CA) and imaged.

Multichannel recording of neuronal activity. To record the neuronal activity, we used the MED64 System, an $8 \times 8$ planar electrode array connected to a 64-channel amplifier (Alpha MED Sciences, Tokyo, Japan). The neuronal activity from an SCN slice was continuously recorded at $37^{\circ} \mathrm{C}$ (sampling rate: $20 \mathrm{kHz}$; low-pass filter: $10 \mathrm{kHz}$; high-pass filter: $100 \mathrm{~Hz}$; amplification: 1000). Spike detector software (Alpha MED Sciences) was used to isolate a spontaneous discharge from the background noise. The number of spikes was counted every $10 \mathrm{~min}$. The data sets were detrended by subtracting the $24 \mathrm{~h}$ running average from the raw data and, thus, the circadian peaks of the SCN firing rate became clearly distinguishable. Our measuring procedure is not suitable for detecting the circadian troughs of the firing frequency, because only the spike signals higher than a fixed threshold were counted.

\section{Results}

To address the role of MAPK in regulating circadian rhythms, we generated transgenic mice carrying an hBmall-luciferase construct to measure clock-gene activity in real time in the SCN (Fig. 1A, left). We prepared SCN slice cultures, and measured the bioluminescence with a photomultiplier tube. We successfully confirmed circadian transcription of Bmal1 over a period of 1-2 weeks. Next, to examine the role of MAPK in circadian gene expression in the SCN, SCN slice cultures were treated for $2 \mathrm{~d}$ with 1,4-diamino-2,3-dicyano-1,4-bis(o-aminophenylmercapto) butadiene (U0126), a specific inhibitor of the MAPK direct activator ERK kinase (MEK) (Fig. $1 A$, right). We confirmed previously that U0126 does not affect luciferase enzyme activity or light emission (data not shown). The pharmacological inactivation of MAPK significantly dampened the rhythms and reduced the basal levels of circadian transcription of Bmall. The detrended data showed that the amplitude was reduced to $\sim 40 \%$ (supplemental Fig. 1, available at www.jneurosci.org as supplemental material). Treatment with the solvent DMSO did not have a significant effect. After washout of U0126, the SCN explants recovered a robust circadian gene expression, demonstrating that these effects were not the result of toxicity. This reversibility also indicates that the rhythm-generating potential in the SCN is preserved even in the presence of U0126.

C Third ventricle.
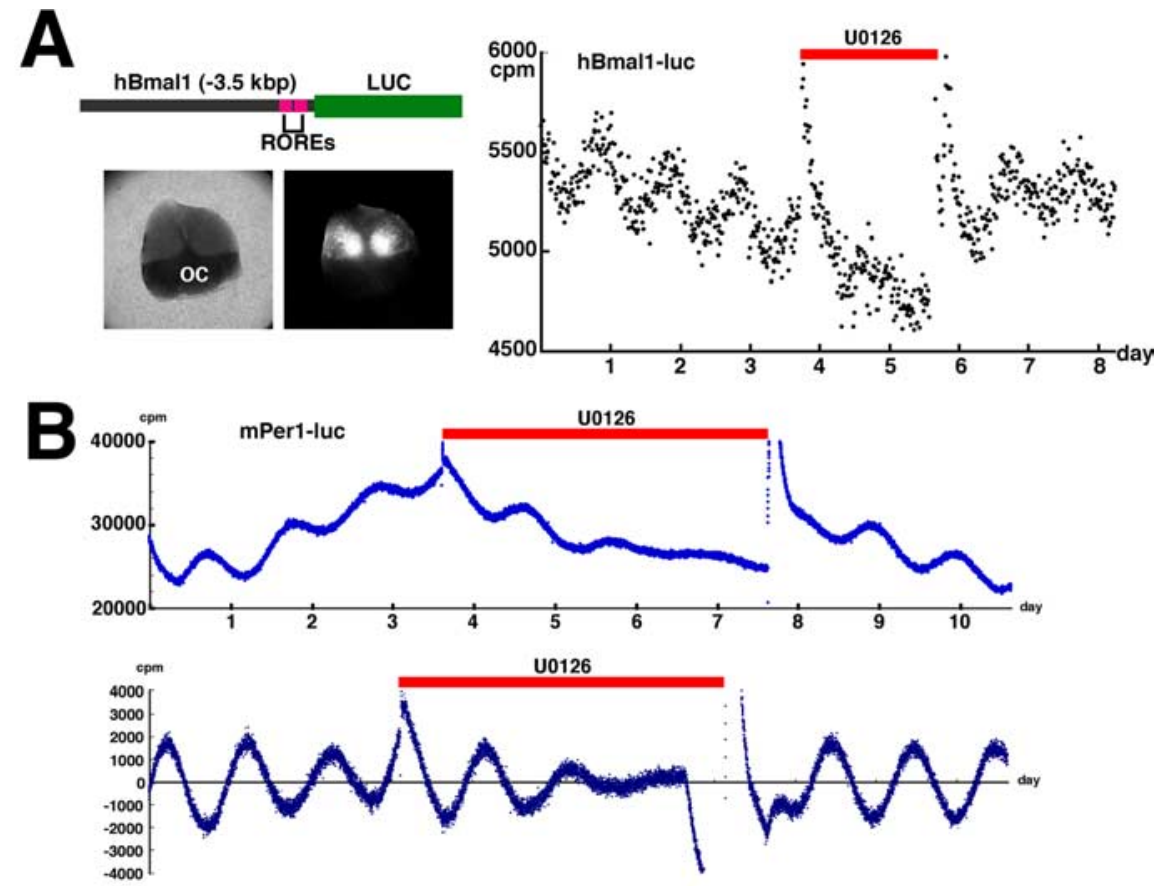

U0126 $(20 \mu \mathrm{M})$
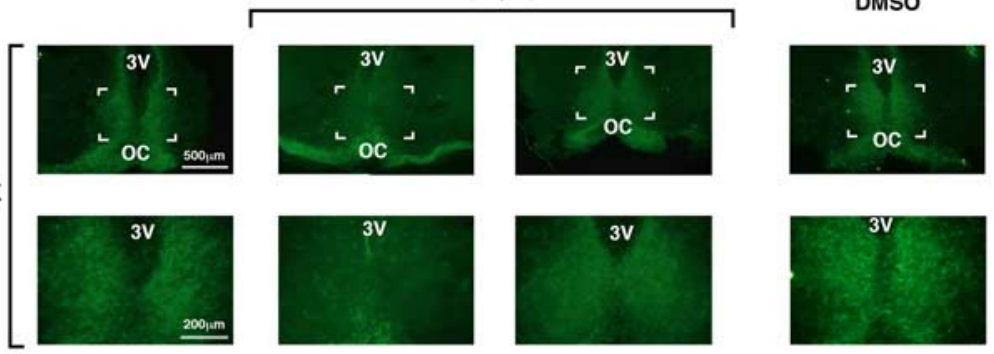

Washout

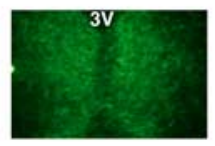

$20 \mathrm{hr}$

Figure 1. The effect of MAPK inhibition on circadian clock gene expression in the SCN. $\boldsymbol{A}$, Left, Diagram of the $h B m a l 1-l u c$ transgene construct, and a brightfield and bioluminescence image of the SCN from an hBmal1-luc transgenic mouse. OC, Optic chiasma; ROREs, ROR/REV-ERB response elements. Right, Paired SCN neurons were placed on a culture membrane, and biolumisimilar results. $\boldsymbol{B}$, The SCN were excised and explanted from an mPer1-luc transgenic rat, and bioluminescence was measured in subtracting the $24 \mathrm{~h}$ running average from the raw data. The experiments were independently repeated three times with a different slice and gave similar results. C, Rat SCN slice cultures were prepared and cultured for 1 week, and immunohistochemistry wasperformed using phospho-p44/42 MAP kinase antibody. The SCN neurons were either left untreated or incubated with U0126 $(20 \mu \mathrm{M})$ or DMSO for $20 \mathrm{~h}$ and then fixed. For the washout condition, U0126 $(20 \mu \mathrm{m})$ was washed out from the SCN after $48 \mathrm{~h}$ and the SCN neurons were incubated for an additional $48 \mathrm{~h}$ before fixation. The boxed areas in the top correspond to the bottom. $3 \mathrm{~V}$,

The transcription of Bmall is positively and negatively regulated by the nuclear orphan receptors retinoid-related orphan receptor $\alpha(\mathrm{ROR} \alpha)$ and $\mathrm{REV}-\mathrm{ERB} \alpha$, respectively (Preitner et al., 2002). To examine whether MAPK inhibition affects clock genes whose transcription is regulated by a different mechanism, similar experiments were performed using SCN slice cultures prepared from Period1-luciferase transgenic rats (Yamazaki et al., 2000) (Fig. 1B). The expression of Period (Per) genes is driven by the CLOCK [neuronal PAS domain protein 2 (NPAS2)]/BMAL1 transcription complex, and PER proteins, together with Cryptochrome, feed back to negatively regulate the CLOCK (NPAS2)/ BMAL1 transcription complex (Ko and Takahashi, 2006). As in the case of Bmall transcription, the inactivation of MAPK dampened the rhythms and reduced the basal levels in the circadian 

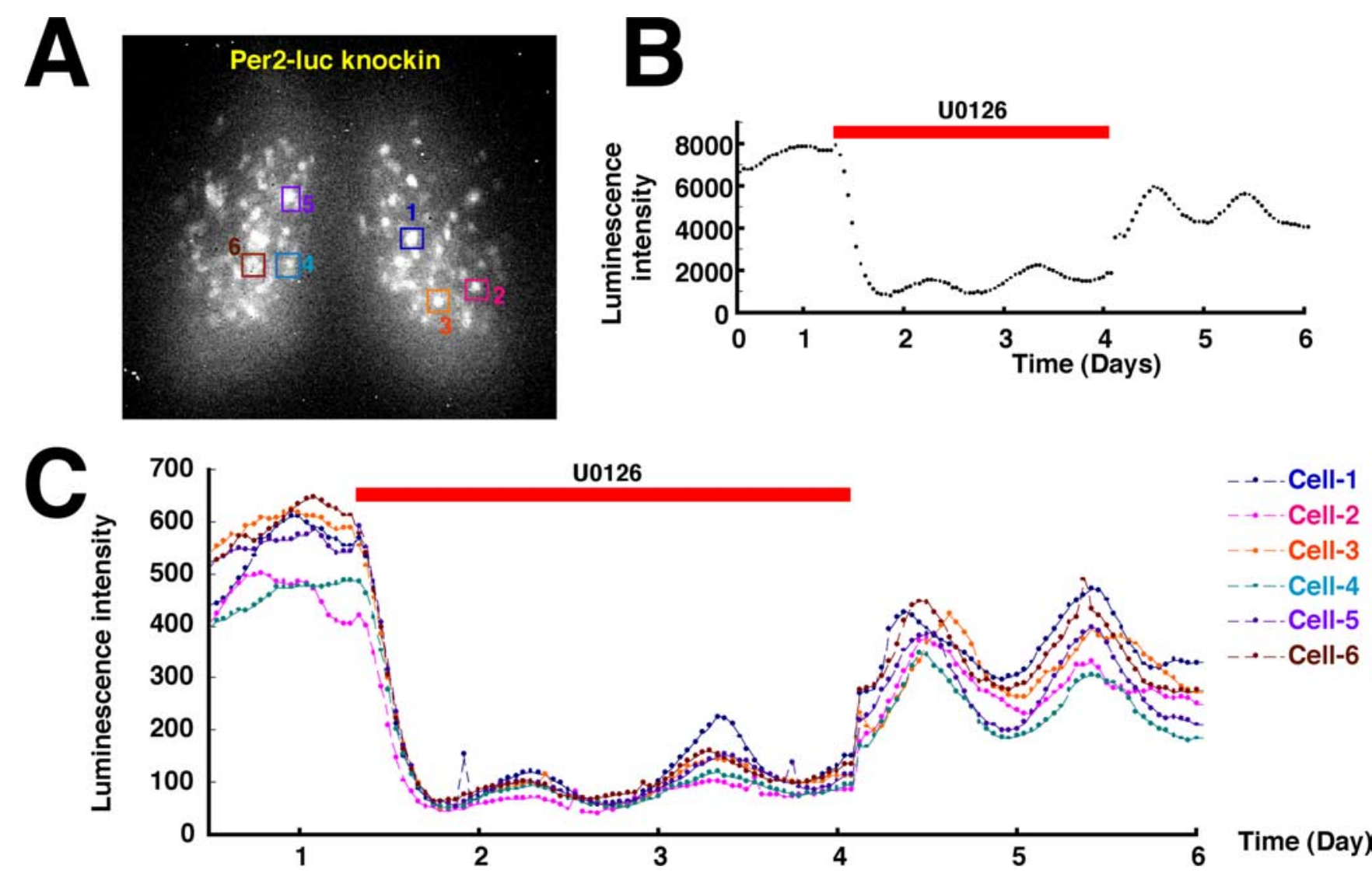

Figure 2. The role of MAPK in individual SCN cellular clocks. A, A bioluminescence image of the SCN from a Per2-luciferase knock-in mouse. Square boxes indicate single SCN neurons whose bioluminescence was monitored. $\boldsymbol{B}$, The total luminescence intensity of the $S C N$ was measured in real time with a highly sensitive cryogenic $C C D$ camera. U0126 (20 $\mu \mathrm{m})$ treatment was performed for $\sim 65 \mathrm{~h}$ (red bar). $\boldsymbol{C}$, The luminescence intensity from individual $\mathrm{SCN}$ neurons was measured in real time. The same sample as in $\boldsymbol{B}$ was analyzed at the single neuron level. The experiments were independently repeated four times with a different slice and gave similar results.

transcription of Per1. Similar results were also obtained with Per2-luciferase knock-in mice (Fig. 2 B). The washout of U0126 reinstated the robust circadian transcription of both Per1 and Per2.

In addition, we were able to immunohistochemically detect active MAPK in the ex vivo SCN slice (Fig. $1 C$ ). However, active MAPK immunoreactivity was almost undetectable in the presence of U0126 and, importantly, the MAPK activity was restored by the removal of the drug, consistent with the restoration of circadian gene expression observed in Figures $1 A, B$, and $2 B$. Together, these data suggest that MAPK is autonomously activated in an ex vivo SCN slice, where it is involved in the maintenance of a robust circadian gene expression. The MAPK activity in the rat SCN oscillates with a similar phase as that in mice (Guillaumond et al., 2007), and, therefore, we used the rat SCN for a higher frequency of similar slice surfaces. However, we could not exclude the possibility that MAPK plays different roles between these species, because MAPK activity shows a different anatomical distribution between the mouse and rat SCN. Future studies are required to determine the species difference in the MAPK role. We were also unable to conclusively determine whether levels of activated MAPK showed circadian fluctuations in the ex vivo SCN slice immunohistochemically, as it was difficult to quantify the signal intensity because of the explant thickness $(400 \mu \mathrm{m})$ and variable slice surface. Previous reports have demonstrated that two rhythmic regions of phospho-p44/42 MAPK (pMAPK) immunoreactivity are observed in the hamster and mouse SCN: a shell-like pattern with peak expression during subjective day and a core-like pattern present during subjective night (Obrietan et al., 1998; Lee et al., 2003). Unfortunately, we cannot discuss the detailed anatomical distribution of PMAPK in experiments using cultured SCN slices because of faint images and ambiguous slice surfaces

Counting the total luciferase activity from the cultured SCN does not allow us to determine what happens to individual cellular clocks. To examine the effects of MAPK inactivation on circadian gene expression in individual neurons, we performed a single-cell bioluminescence imaging of an ex vivo SCN slice prepared from Per2-luciferase knock-in mice (Yoo et al., 2004) (Fig. $2 A)$. By using knock-in mice expressing PER2-LUC fusion protein from the endogenous Per2 genomic locus, we can exclude indirect or secondary effects on transgene transcription caused by the genomic location, incomplete promoter/enhancer activation, or transgene copy number (these can all be potential problems in the case of transgenic mice). Therefore, the luciferase activity from the Per2-luciferase knock-in mice will accurately reflect the endogenous transcriptional regulation of Per2. The bioluminescence was measured in real-time in several individual cells with a highly sensitive cryogenic CCD camera (LV200; Olympus). The cultured SCN neurons were treated with U0126 for $2 \mathrm{~d}$, after which the U0126 was washed away (Fig. $2 C$ ). Figure $2 B$ represents the total bioluminescence from SCN cultures. The specific inhibition of the MAPK activity resulted in an immediate and drastic decrease of the basal PER2-LUC expression in individual neurons (supplemental Fig. 2, available at www.jneurosci.org as supplemental material). The circadian oscillation of PER2-LUC 
expression was not completely abolished, but was significantly dampened. Treatment with the solvent DMSO did not have a significant effect. After washout of the U0126, the basal expression levels and oscillation amplitude of PER2-LUC were gradually restored, as observed in the hBmal1-luc transgenic mice in Figure $1 \mathrm{~A}$. Although slight variations in the extent to which the basal levels and oscillation amplitude of Per 2 transcription decreased was observed among the individual cells, all of the cells measured in this experiment showed a similar disturbance in the circadian oscillation of the Per 2 expression in response to U0126. There are two possible interpretations of these observations: one is that the activity of MAPK is involved in circadian gene expression in every SCN neuron, and the other is that MAPK plays a role in circadian gene expression in a specific subset of SCN neurons and the effects derived from the inactivation of MAPK in these neurons spread over the entire SCN through intercellular communication. According to previous reports, MAPK is an intracellular mediator of both the VIP (vasoactive intestinal polypeptide) and AVP (arginine vasopressin) signaling (Arima et al., 2002; Hughes et al., 2004), implying another possibility that the effects of MAPK inhibition on Per2 transcription result from disruption of the intercellular communication among SCN neurons. In each experiment, we monitored $>15$ signals, in addition to the six cells shown which are easily and reliably distinguishable as a single cell. However, we still could not conclude that MAPK activity is required for Per2 circadian transcription in all the individual SCN neurons.

SCN neuronal activity exhibits autonomous circadian rhythms, which are thought to play an indispensable role in the synchronization of individual SCN cellular clocks and the generation of behavioral and physiological output rhythms. Moreover, a previous study suggests that the SCN firing rhythms contribute to the maintenance of the robust circadian expression of the clock genes in the SCN (Yamaguchi et al., 2003). However, the molecular mechanism by which circadian firing rhythms interact with circadian clock gene expression remains undefined. We investigated whether inactivation of MAPK affects the rhythm of SCN neuronal activity. An SCN slice was cultured on a multipleelectrode array, and multiunit activity was continuously recorded. The neuronal firing signals were extracted from the raw voltage traces by using Spike Detector software (Fig. 3A), and the number of spikes was counted every $10 \mathrm{~min}$ (Fig. $3 B, C$, each representing an individual experiment). Before the addition of U0126, the firing activity showed a circadian pattern as reported previously (Welsh et al., 1995). The detrended data unmasked the circadian peaks of the SCN neuronal activity (supplemental Fig. $3 A, B$, available at www.jneurosci.org as supplemental material). The day 2 peak was hardly affected by the U0126 addition, whereas the subsequent peak (day 3 ) was almost completely abolished, at least during the U0126 treatment (supplemental Fig. $3 C$, available at www.jneurosci.org as supplemental material). Although a transient firing surge just after the removal of U0126 was thought to be a temporary response induced by sample han-


Time (Day)

Figure 3. Involvement of MAPK in suprachiasmatic firing rhythms. $\boldsymbol{A}$, Representative data of raw voltage traces are shown.

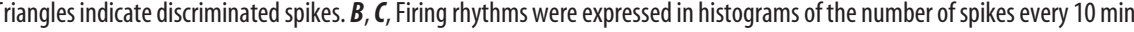
experiments were independently repeated four times with a different slice and gave similar results. The individual data show single-electrode derived signals and were obtained from different SCN slices.

dling, we still cannot exclude the possibility that it may be the day 3 peak, phase-delayed by U0126. Treatment with the solvent DMSO did not have a significant effect. This slow response of firing activity to MAPK inhibition is significantly different from the relatively rapid response of clock gene expression to MAPK inhibition observed in Figures 1 and 2. One possible interpretation of this data are that the MAPK signaling cascade may be both a downstream target of the neuronal discharge and an upstream mediator of clock gene expression. Therefore, MAPK inhibition initially disturbs the circadian clock gene expression, and after a time lag, this disturbance is reflected as the severe abolishment of the firing rhythms. The removal of the drug from the SCN slice cultures restored the robust rhythmic firing, albeit with a different phase from the original rhythm, as observed for circadian clock gene expression (Fig. $1 A, B, 2 B$ ). However, the restoration of the rhythmic firing by removal of the drug was not infrequently undetectable. Unfortunately, we could not distinguish whether this was because cultured tissues were broken off from the electrodes by sample handling such as pipetting, or because the rhythmic firing was irreversibly abolished by U0126. There appears to be some degree of variation among individual neurons or mice in the effect of U0126 on firing rhythms, suggesting that the sensitivity of individual SCN neurons to the MEK inhibitor may be not homogeneous. For example, the day 2 peak of firing rhythms in Figure 3B was not affected by the presence of U0126, whereas the corresponding peak in Figure $3 C$ was severely phaseshifted. Together, these data demonstrate that MAPK activity plays a pivotal role in the suprachiasmatic firing rhythms, as well as in the circadian clock gene expression.

\section{Discussion}

In several other organisms, the circadian fluctuation of the MAPK activity in the circadian pacemaker is involved in circa- 
dian input pathways (Cermakian et al., 2002) or behavioral/physiological outputs (Sanada et al., 2000; Ko et al., 2001; Williams et al., 2001), but there have been no reports indicating that MAPK functions as a core clock component. Our results demonstrate that activated MAPK is significantly detectable in an ex vivo SCN slice (morphological differences among species remain undefined), and suggest an essential role for MAPK activity in the maintenance of robust circadian clock gene expression in individual neurons. We also found that MAPK is involved in regulating the robustness of suprachiasmatic firing rhythms. Although several studies have reported that regional differences in MAPK activity rhythms are observed (Obrietan et al., 1998; Lee et al., 2003), our single-cell imaging studies imply that SCN neurons located in any compartment may require MAPK activity for robust circadian rhythms. Thus, these results strongly suggest that MAPK is a functional component in the SCN autonomous circadian system. Light-induced discharges in the SCN neurons may activate MAPK (Obrietan et al., 1999; Tischkau et al., 2000), thus implying the possibility that light-independent circadian discharges also function as upstream signals to activate MAPK in a circadian manner. The cAMP response element binding protein (CREB) is generally believed to be one of the downstream targets of activated MAPK in the SCN (Obrietan et al., 1999; Coogan and Piggins, 2004), and activated CREB may drive circadian transcription of clock genes such as Per1 (Travnickova-Bendova et al., 2002). Both neuronal activity rhythms and circadian clock gene expression have been thought to be necessary for well synchronized and robust autonomous circadian rhythms in the SCN, and one possible interpretation is that these two components of the SCN autonomous circadian system are functionally interconnected via the MAPK signaling cascade.

\section{References}

Arima H, House SB, Gainer H, Aguilera G (2002) Neuronal activity is required for the circadian rhythm of vasopressin gene transcription in the suprachiasmatic nucleus in vitro. Endocrinology 143:4165-4171.

Cermakian N, Pando MP, Thompson CL, Pinchak AB, Selby CP, Gutierrez L, Wells DE, Cahill GM, Sancar A, Sassone-Corsi P (2002) Light induction of a vertebrate clock gene involves signaling through blue-light receptors and MAP kinases. Curr Biol 12:844-848.

Coogan AN, Piggins HD (2004) MAP kinases in the mammalian circadian system - key regulators of clock function. J Neurochem 90:769-775.

Dunlap JC (1999) Molecular bases for circadian clocks. Cell 96:271-290.

Guillaumond F, Becquet D, Blanchard MP, Attia J, Moreno M, Bosler O, Francois-Bellan AM (2007) Nocturnal expression of phosphorylatedERK1/2 in gastrin-releasing peptide neurons of the rat suprachiasmatic nucleus. J Neurochem 101:1224-1235.

Hughes AT, Fahey B, Cutler DJ, Coogan AN, Piggins HD (2004) Aberrant gating of photic input to the suprachiasmatic circadian pacemaker of mice lacking the VPAC2 receptor. J Neurosci 24:3522-3526.

Ko CH, Takahashi JS (2006) Molecular components of the mammalian circadian clock. Hum Mol Genet 15:R271-R277.

Ko GY, Ko ML, Dryer SE (2001) Circadian regulation of cGMP-gated cationic channels of chick retinal cones. Erk MAP Kinase and $\mathrm{Ca}^{2+}$ / calmodulin-dependent protein kinase II. Neuron 29:255-266.

Lee HS, Nelms JL, Nguyen M, Silver R, Lehman MN (2003) The eye is necessary for a circadian rhythm in the suprachiasmatic nucleus. Nat Neurosci 6:111-112.

Obrietan K, Impey S, Storm DR (1998) Light and circadian rhythmicity regulate MAP kinase activation in the suprachiasmatic nuclei. Nat Neurosci 1:693-700.

Obrietan K, Impey S, Smith D, Athos J, Storm DR (1999) Circadian regulation of cAMP response element-mediated gene expression in the suprachiasmatic nuclei. J Biol Chem 274:17748-17756.

Preitner N, Damiola F, Lopez-Molina L, Zakany J, Duboule D, Albrecht U, Schibler U (2002) The orphan nuclear receptor REV-ERBalpha controls circadian transcription within the positive limb of the mammalian circadian oscillator. Cell 110:251-260.

Reppert SM, Weaver DR (2002) Coordination of circadian timing in mammals. Nature 418:935-941.

Sanada K, Hayashi Y, Harada Y, Okano T, Fukada Y (2000) Role of circadian activation of mitogen-activated protein kinase in chick pineal clock oscillation. J Neurosci 20:986-991.

Schibler U, Sassone-Corsi P (2002) A web of circadian pacemakers. Cell 111:919-922.

Tischkau SA, Gallman EA, Buchanan GF, Gillette MU (2000) Differential cAMP gating of glutamatergic signaling regulates long-term state changes in the suprachiasmatic circadian clock. J Neurosci 20:7830-7837.

Travnickova-Bendova Z, Cermakian N, Reppert SM, Sassone-Corsi P (2002) Bimodal regulation of mPeriod promoters by CREB-dependent signaling and CLOCK/BMAL1 activity. Proc Natl Acad Sci USA 99:7728-7733.

Wang GK, Sehgal A (2002) Signaling components that drive circadian rhythms. Curr Opin Neurobiol 12:331-338.

Welsh DK, Logothetis DE, Meister M, Reppert SM (1995) Individual neurons dissociated from rat suprachiasmatic nucleus express independently phased circadian firing rhythms. Neuron 14:697-706.

Williams JA, Su HS, Bernards A, Field J, Sehgal A (2001) A circadian output in Drosophila mediated by neurofibromatosis-1 and Ras/MAPK. Science 293:2251-2256.

Yamaguchi S, Isejima H, Matsuo T, Okura R, Yagita K, Kobayashi M, Okamura H (2003) Synchronization of cellular clocks in the suprachiasmatic nucleus. Science 302:1408-1412.

Yamazaki S, Numano R, Abe M, Hida A, Takahashi R, Ueda M, Block GD, Sakaki Y, Menaker M, Tei H (2000) Resetting central and peripheral circadian oscillators in transgenic rats. Science 288:682-685.

Yoo SH, Yamazaki S, Lowrey PL, Shimomura K, Ko CH, Buhr ED, Siepka SM, Hong HK, Oh WJ, Yoo OJ, Menaker M, Takahashi JS (2004) PERIOD2::LUCIFERASE real-time reporting of circadian dynamics reveals persistent circadian oscillations in mouse peripheral tissues. Proc Natl Acad Sci USA 101:5339-5346.

Young MW, Kay SA (2001) Time zones: a comparative genetics of circadian clocks. Nat Rev Genet 2:702-715. 